\title{
Decoherence of Polaron in Asymmetric Quantum Dot Qubit Under an Electromagnetic Field
}

\author{
Alain Jerve Fotue ${ }^{1,}$, , Sadem Christian Kenfack ${ }^{1}$, Nsangou Issofa ${ }^{1}$, Maurice Tiotsop ${ }^{1}$, \\ Michel Pascal Tabue Djemmo ${ }^{1,3}$, Amos Veyongni Wirngo ${ }^{1}$, Hilaire Fotsin ${ }^{2}$, Lukong Cornelius Fai ${ }^{1}$ \\ ${ }^{1}$ Mesoscopic and Multilayers Structures Laboratory, Department of Physics, Faculty of Science, University of Dschang, Dschang, Cameroon \\ ${ }^{2}$ Laboratory of Electronics and Signal Processing, Department of Physics, Faculty of Science, University of Dschang, Dschang, Cameroon \\ ${ }^{3}$ Laboratory of Mechanics and Modeling of Physical Systems, Faculty of Science, University of Dschang, Dschang, Cameroon
}

Email address:

fotuea@yahoo.fr (A. J. Fotue)

\section{To cite this article:}

Alain Jerve Fotue, Sadem Christian Kenfack, Nsangou Issofa, Maurice Tiotsop, Michel Pascal Tabue Djemmo, Amos Veyongni Wirngo, Hilaire Fotsin, Lukong Cornelius Fai. Decoherence of Polaron in Asymmetric Quantum Dot Qubit Under an Electromagnetic Field. American Journal of Modern Physics. Vol. 4, No. 3, 2015, pp. 138-148. doi: 10.11648/j.ajmp.20150403.16

\begin{abstract}
In this paper, we investigate the time evolution of the quantum mechanical state of a polaron using the Pekar type variational method on the electric-LO-phonon and the magnetic-LO-phonon strong coupling in a quantum dot. We obtain the Eigen energies and the Eigen functions of the ground state and the first excited state, respectively. In a quantum dot, this system can be viewed as a two level quantum system qubit. The superposition state polaron density oscillates in the quantum dot with a period $\tau_{0}$ when the polaron is in the superposition of the ground and the first-excited states. The spontaneous emission of phonons causes the decoherence of the qubit. We show that the density matrix of the qubit decays with the time while the coherence term of the density matrix element $p_{01}$ ( or $p_{10}$ ) decays with the time as well for different coupling strengths, confinement lengths, and dispersion coefficients. The Shannon entropy is evaluated in order to investigate the decoherence of the system.
\end{abstract}

Keywords: Polaron, Quantum Dot, Qubit, Electric Field, Magnetic Field, Cyclotron Frequency, Shannon Entropy, Decoherence

\section{Introduction}

With the exponential advancement of nanotechnology during these last years, the study of quantum computing and quantum information processing has generated widespread interest. The two-level system is usually employed as the elementary unit for storing information. Quantum computation will be based on the laws of quantum mechanics Several schemes have been proposed for realizing quantum computers in recent years [1-9]. For quantum computers to have an edge over classical computers, they will need to carry thousands of qubits. Consequently, these quantum computers with large numbers of qubits will be most feasible as solid-state systems. Quantum computation is a process of quantization and it is necessary to maintain the superposition of quantum states to some extent in the entire computing process. However, quantum systems are very frail and the interaction of a quantum memory with its environment destroys the quantum coherence of the stored information, a process called decoherence. This necessitates the proper isolation of a qubit from its external environment Therefore, quantum decoherence plays a very important role in the formalism of quantum computing. Considerable effort has been made in recent years to investigate quantum decoherence and how to prolong the decoherence time [10 14]. Coherent manipulation and storage of quantum information are required in order to construct a working quantum computer and rely on reducing decohering interaction between its basic elements, the qubits, and their environment.

After the pioneering work on the information theory by Shannon [15], many studies have been carried out on the question of how information storage, processing and transmission tasks can be performed with macroscopic decohered resources [16,17]. In this light, self-assembled quantum dots (QDs) have attracted substantial attention due to their perfect crystal structures. Therefore, the realization of qubits using solid state devices has become one of the most popular solid-state quantum information research fields. 
Many schemes have been proposed for carrying out research on decoherence in quantum nanostructures $[1,2,19]$, but an investigation of decoherence in an asymmetrical quantum dot, taking into consideration, electric and magnetic fields has not yet been carried out. The spontaneous emission of phonons causes decoherence of the qubit. We show the decay of the density matrix of the qubit during its evolution with time and discuss the relations between the coherence term of the density matrix element $p_{01}\left(\right.$ or $\left.p_{10}\right)$ and the coupling strength, the confinement length and the dispersion coefficent $[10,20$ 22]

This article is organized as follows: in section 2, the theory and calculations are presented Therein, we derive the Eigen energies and Eigen functions of the ground and first excited states of the polaron in a cylindrical QD using the Pekar variational method and considering the potential in the electron-LO-phonon strong coupling. We obtain the probability density of the polaron which oscillates with a given period when it is in a superposition of the ground and first excited states. The expressions relating the period of oscillation and the transition frequency of the polaron to the cyclotron frequency and the electric field density parameter are derived. The Shannon entropy is also calculated. In section 3, numerical results are presented and discussed. The last section is devoted to the conclusion

\section{Theory and Calculation}

We consider a system in which the electrons are moving in a polar crystal quantum dot with a three-dimensional harmonic potential and interacting with bulk LO phonons under the influence of an electric and a magnetic field. The electric field $F$ is along the $\rho$ - direction while the magnetic field is along the $z$ - direction with vector potential $A=B\left(-\frac{y}{2}, x / 2,0\right)$. The Hamiltonian of the electron-phonon interaction system can be written as:

$$
\begin{aligned}
H & =\frac{1}{2 m}\left(p_{x}-\frac{\beta^{2}}{4} y\right)^{2}+\frac{1}{2 m}\left(p_{y}+\frac{\beta^{2}}{4} x\right)^{2}-e^{*} \rho F+\frac{p_{z}^{2}}{2 m}+ \\
& +\sum_{q} \hbar \omega_{L O} a_{q}^{+} a_{q}+\frac{1}{2} m \omega_{1}^{2} \rho^{2}+\frac{1}{2} m \omega_{2}^{2} z^{2}+\sum_{q}\left[V_{q} a_{q} \exp (\text { iqr })+\text { h.c. }\right] .
\end{aligned}
$$

$\beta^{2}=(2 e / c) B$ where $c$ is the speed of light in vacuum and $m$ is the band mass while $\omega_{1}$ and $\omega_{2}$ are the magnitudes of the transverse and longitudinal confinement strengths of the potentials in the $x-y-$ plane and the $z-$ direction, respectively. $a_{q}^{+}\left(a_{q}\right)$ denotes the creation (annihilation) operator of the bulk $L O$ phonon with the wave vector $q$, $p=\left(p_{x}, p_{y}, p_{z}\right)$ and $r=(\rho, z)$ are the momentum and position vectors of the electron and $\rho=(x, y)$ is the position vector of the electron in the $x-y-$ plane. $V_{q}$ and $\alpha$ in (2.1) are given as follows:

$$
\begin{aligned}
& V_{q}=i\left(\frac{\hbar \omega_{L O}}{q}\right)\left(\frac{\hbar}{2 m \omega_{L O}}\right)^{1 / 4}\left(\frac{4 \pi}{V}\right)^{1 / 2} \\
& \alpha=\left(\frac{e^{2}}{2 \hbar \omega_{L O}}\right)\left(\frac{2 m \omega_{L O}}{\hbar}\right)^{1 / 2}\left(\frac{1}{\varepsilon_{\infty}}-\frac{1}{\varepsilon_{0}}\right) .
\end{aligned}
$$

To evaluate the energy of our polaron, we use the Pekar variational method. To achieve our goal, we choose the trial function of strong -coupling that can be separated into two parts which individually describe the electron and the phonon. The trial function drawn from [15] is written as:

$$
|\psi\rangle=|\phi\rangle U\left|0_{p h}\right\rangle
$$

Where $|\phi\rangle$ depends only on the electron coordinate, $\left|0_{p h}\right\rangle$ represents the phonon's vacuum state with $a_{q}\left|0_{p h}\right\rangle=0$, and
$U\left|0_{p h}\right\rangle$ is the coherent state of the phonon,

$$
U=\exp \left[\sum_{q}\left(a_{q}^{+} f_{q}-a_{q} f_{q}^{*}\right)\right]
$$

where $f_{q}\left(f_{q}^{*}\right)$ is a variational function. We may choose the trial ground and the first-excited state wave functions of the electron to be

$$
\begin{aligned}
& \left|\phi_{0}\right\rangle=\left(\frac{2 \lambda_{0}}{\pi}\right)\left(\frac{\mu_{0}}{\pi}\right) \exp \left[-\frac{\lambda_{0} \rho^{2}}{2}\right] \exp \left[-\frac{\mu_{0} z^{2}}{2}\right], \\
& \left|\phi_{1}\right\rangle=2\left(\frac{\lambda_{1}}{\pi}\right)^{1 / 2} \frac{\mu_{1}^{3 / 4}}{\pi^{1 / 4}} \exp \left[-\frac{\lambda_{1} \rho^{2}}{2}\right] \exp \left[-\frac{\mu_{1} z^{2}}{2}\right] z
\end{aligned}
$$

where $\lambda_{0}, \mu_{0}, \lambda_{1}$ and $\mu_{1}$ are the variational parameters. Equations (2.5) and (2.6) satisfy the following normalized relations:

$$
\left\langle\phi_{0} \mid \phi_{0}\right\rangle=\left\langle\phi_{1} \mid \phi_{1}\right\rangle=1 ;\left\langle\phi_{0} \mid \phi_{1}\right\rangle=0
$$

Using the Pekar variational method, we have

$$
H^{\prime}=U^{-1} H U
$$

By minimizing the expectation value of the Hamiltonian, that is $E_{0}=\left\langle\phi_{0}\left|H^{\prime}\right| \phi_{0}\right\rangle$ and $E_{1}=\left\langle\phi_{1}\left|H^{\prime}\right| \phi_{1}\right\rangle$, we then obtain the magnetopolaron ground and first excited state energy in 
the following forms:

$$
E_{0}=\lambda_{0}+\frac{\mu_{0}}{2}+\frac{1}{\lambda_{0} l_{1}^{4}}+\frac{1}{2 \mu_{0} l_{2}^{4}}+\frac{\omega_{c}^{2}}{16 \lambda_{0}}-\frac{\sqrt{\pi} e^{*} F}{2 \sqrt{\mu_{0}}}-\alpha \sqrt{\frac{2 \lambda_{0}}{\pi\left(1-\frac{\lambda_{0}}{\mu_{0}}\right)}} \arcsin \left(1-\frac{\lambda_{0}}{\mu_{0}}\right)^{1 / 2}
$$

and

$$
E_{1}=\lambda_{1}+\frac{3 \mu_{1}}{2}+\frac{1}{\lambda_{1} l_{1}^{4}}+\frac{1}{2 \mu_{1} l_{2}^{4}}+\frac{\omega_{c}^{2}}{16 \lambda_{1}}-\frac{\sqrt{\pi} e^{*} F}{2 \sqrt{\mu_{1}}}-\alpha \sqrt{\frac{2 \lambda_{1}}{\pi\left(1-\frac{\lambda_{1}}{\mu_{1}}\right)}} \arcsin \left(1-\frac{\lambda_{1}}{\mu_{1}}\right)^{1 / 2}
$$

where $l_{1}=\sqrt{\frac{\hbar}{m \omega_{1}}}, l_{2}=\sqrt{\frac{\hbar}{m \omega_{2}}}$ are the transverse and longitudinal effective confinement lengths of the QD, respectively.

The superposition state of the electron can be expressed as:

$$
\left|\psi_{01}\right\rangle=\frac{1}{\sqrt{2}}(|0\rangle+|1\rangle)
$$

Where

$$
|0\rangle=\phi_{0}(\rho, z)=\left(\frac{2 \lambda_{0}}{\pi}\right)\left(\frac{\mu_{0}}{\pi}\right) \exp \left[-\frac{\lambda_{0} \rho^{2}}{2}\right] \exp \left[-\frac{\mu_{0} z^{2}}{2}\right]
$$

And

$$
|1\rangle=\phi_{1}(\rho, z)=2\left(\frac{\lambda_{1}}{\pi}\right)^{1 / 2} \frac{\mu_{1}^{3 / 4}}{\pi^{1 / 4}} \exp \left[-\frac{\lambda_{1} \rho^{2}}{2}\right] \exp \left[-\frac{\mu_{1} z^{2}}{2}\right] z .
$$

We define the time evolution of the system as

$$
\psi_{01}(t, \rho, z)=\frac{1}{\sqrt{2}} \phi_{0}(\rho, z) \exp \left(-i \frac{E_{0} t}{\hbar}\right)+\frac{1}{\sqrt{2}} \phi_{1}(\rho, z) \exp \left(-i \frac{E_{1} t}{\hbar}\right)
$$

The density matrix of this qubit can be expressed as [10]

$$
\xi=\left[\begin{array}{ll}
p_{00} & p_{01} \\
p_{10} & p_{11}
\end{array}\right]
$$

where

$$
\begin{aligned}
& p_{00}=\frac{1}{2}\left|\phi_{0}(\rho, z)\right|^{2} ; p_{11}=\frac{1}{2}\left|\phi_{1}(\rho, z)\right|^{2} ; \\
& p_{01}=\frac{1}{2} \phi_{0}^{*}(\rho, z) \phi_{1}^{*}(\rho, z) \text { and } p_{10}=\frac{1}{2} \phi_{1}^{*}(\rho, z) \phi_{0}^{*}(\rho, z)
\end{aligned}
$$

On the condition of low temperature, we introduce the parabolic phonon dispersion $\omega=\omega_{L O}-\eta c q^{2}$. Under the dipole approximation, based on the Fermi Golden Rule, the spontaneous emission rate [23] can be written in the following form [24]:

$$
\Gamma=\frac{e^{2} \omega_{01}^{3}}{2 \pi c^{3} \varepsilon_{0} \hbar^{2} \eta}|\langle 0|r| 1\rangle|^{2}
$$

where $\varepsilon_{0}$ is the vacuum dielectric constant, $\eta$ is the dispersion coefficient, and $\omega_{01}$ is the energy level spacing between $|0\rangle$ and $|1\rangle$. The electron can transit from the firstexcited state to the ground-state because of the spontaneous emission of phonons, that is, the spontaneous emission of phonons causes the decoherence of the qubit and as such, the time evolution of the density matrix of the qubit can be written as [10]

$$
\xi(t, \rho, z)=\left[\begin{array}{cc}
p_{00}+\left(1-e^{-\Gamma t}\right) p_{11} & e^{-\frac{\Gamma}{2} t} p_{01} \\
e^{-\frac{\Gamma}{2} t} p_{10} & e^{-\Gamma t} p_{11}
\end{array}\right]
$$

The Shannon entropy for the state given by the wave function $\psi_{01}(t, \rho, z)$ is [15]

$$
S(t)=\int d z d \rho\left|\psi_{01}(t, \rho, z)\right|^{2} \ln \left|\psi_{01}(t, \rho, z)\right|^{2}
$$




\section{Numerical Results and Discussions}

entropy to the cyclotron frequency and the electric field

The curves which relate the probability density and the

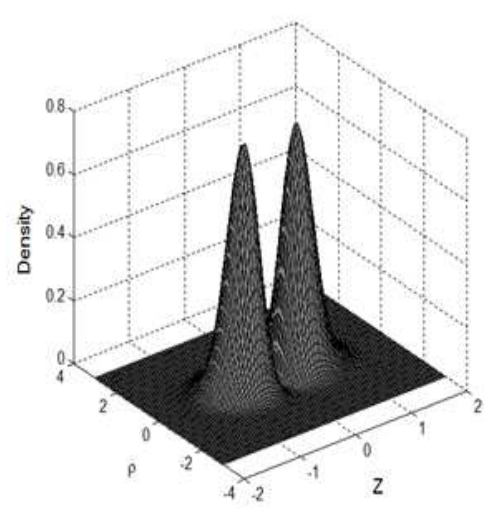

(a)

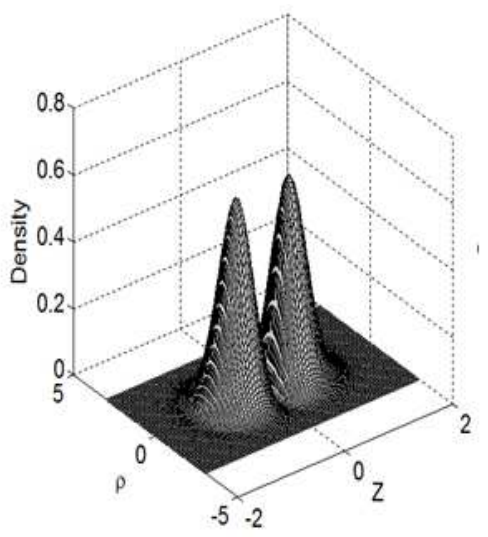

(b)

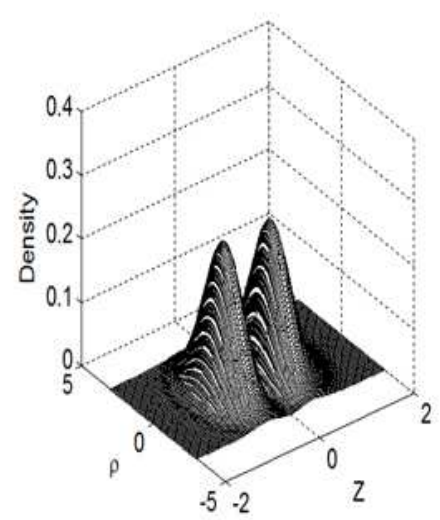

(c)

Figure 1. Probability density when the electron is in superposition state $|0\rangle$ and $|1\rangle$ for $l_{1}=0.75 ; l_{2}=0.5 ; t=1.0 ; F=1.0 ; \alpha=7.0 ; \eta=0.2$, (a) $\omega_{c}=2.7 ;$ (b) $\omega_{c}=5.5 ;$ (c) $\omega_{c}=10.5$

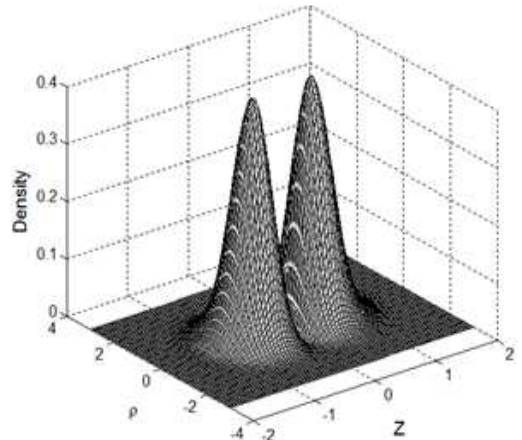

(a)

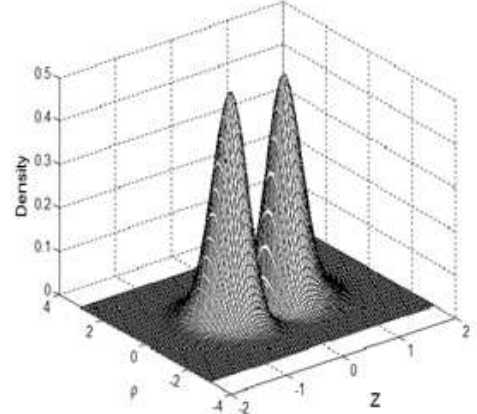

(b)

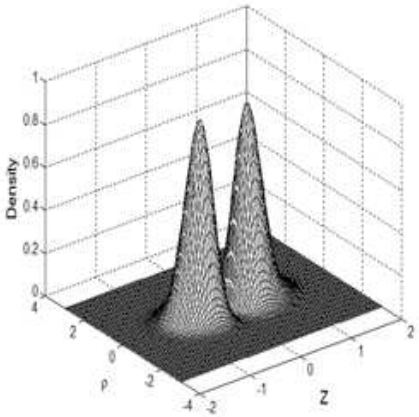

(c)

Figure 2. Probability density when the electron is in superposition state $|0\rangle$ and $|1\rangle$ for $l_{1}=0.75 ; l_{2}=0.5 ; t=1.0 ; \omega_{c}=5.0 ; \alpha=7.0 ; \eta=0.2$, (a) $F=1.5 ;(b)$ $F=3.5$; (c) $F=15.5$.

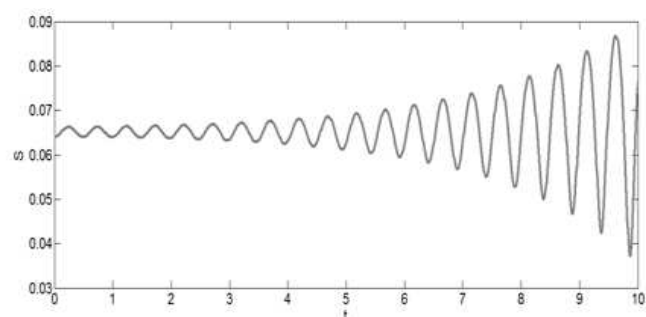

(a)

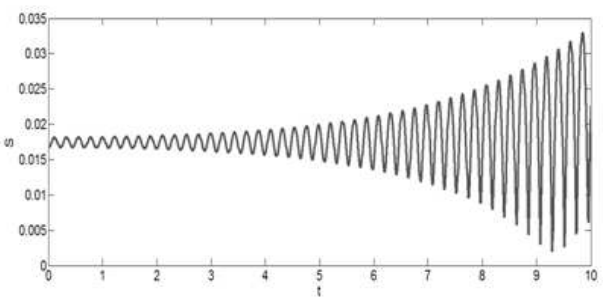

(c)

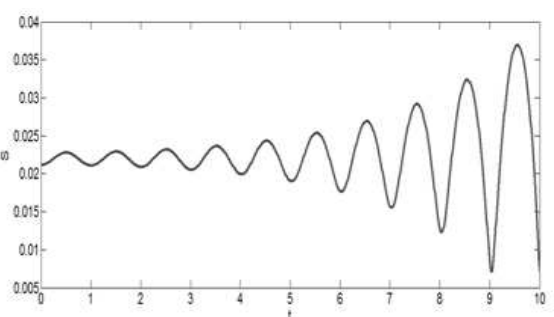

(b)

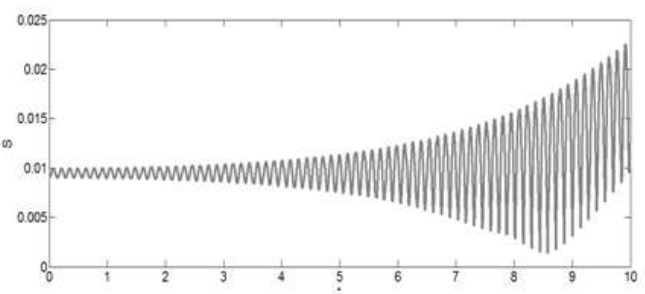

(d)

Figure 3. Time evolution of entropy for $l_{1}=0.75 ; l_{2}=0.5 ; F=1.0 ; \alpha=7.0 ; \eta=0.2$ : (a) $\omega_{c}=1.0 ;$ (b) $\omega_{c}=15.0 ;$ (c) $\omega_{c}=50.0$ and (d) $\omega_{c}=100.0$. 


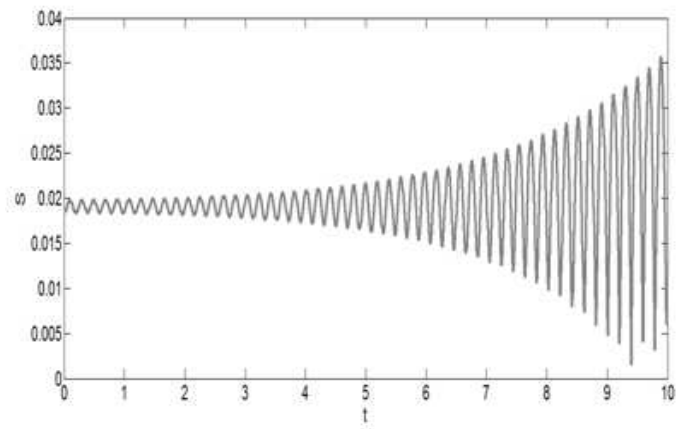

(a)

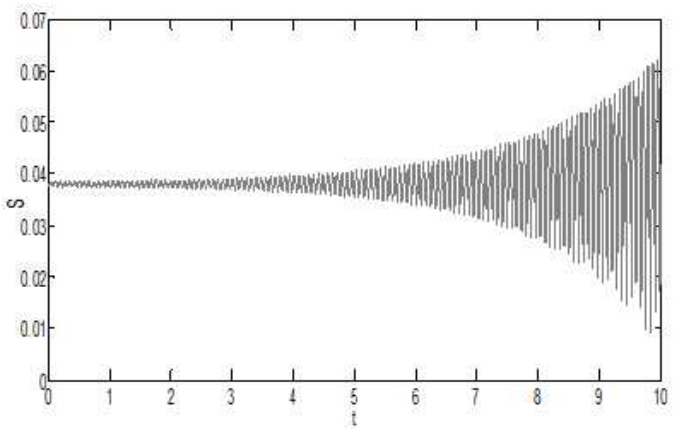

(c)

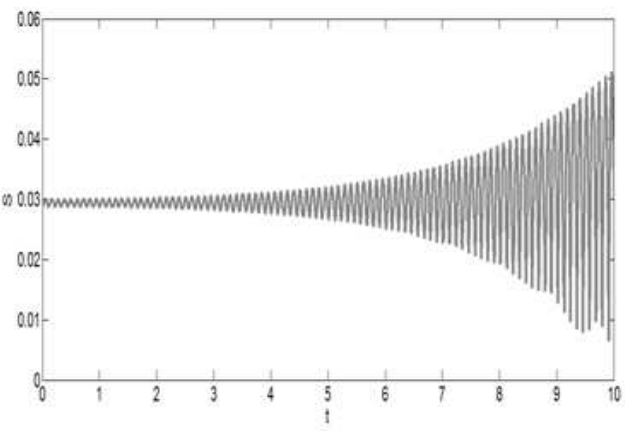

(b)

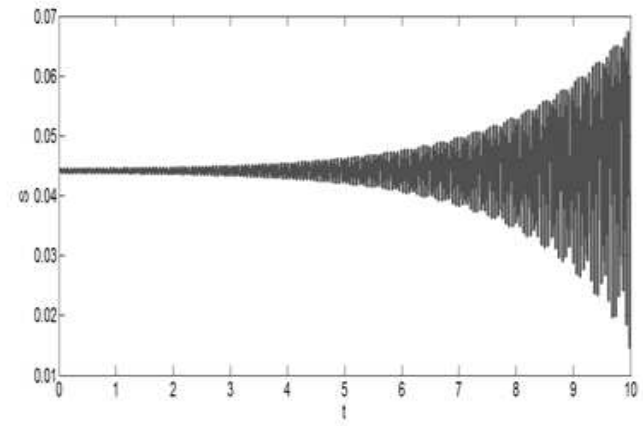

(d)

Figure 4. Time evolution of entropy for $l_{1}=0.75 ; l_{2}=0.5 ; \omega_{c}=50.0 ; \alpha=7.0 ; \eta=0.2$ (a) $F=2.0 ;$ (b) $F=10.0$; (c) $F=20.0$ and (d) $F=30.0$

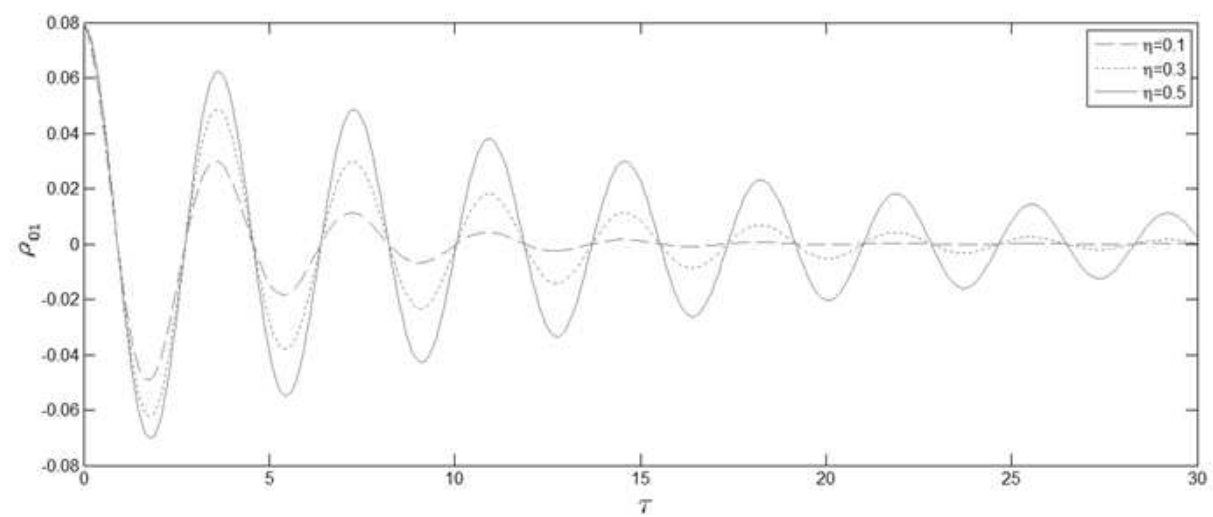

Figure 5. The density matrix elements $p_{01}$ as a function of time $\tau$ for $l_{1}=0.8 ; l_{2}=0.75 ; \alpha=7.0, F=1.5$ and $\omega_{c}=2.0$.

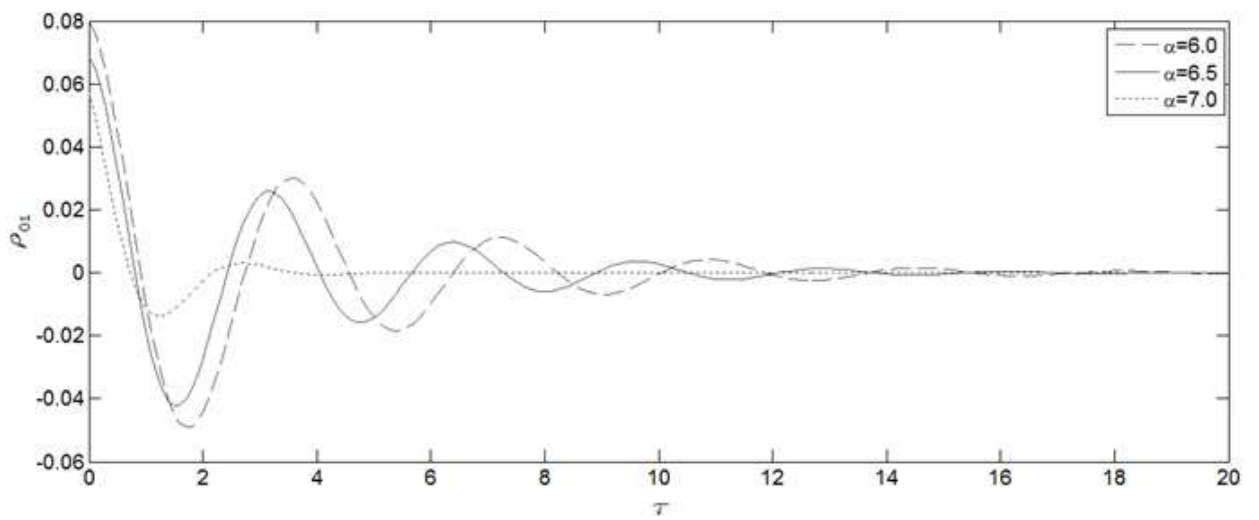

Figure 6. The density matrix elements $p_{01}$ as a function of time $\tau$ for $l_{1}=0.8 ; l_{2}=0.75 ; \eta=0.2, F=1.5$ and $\omega_{c}=2.0$. 


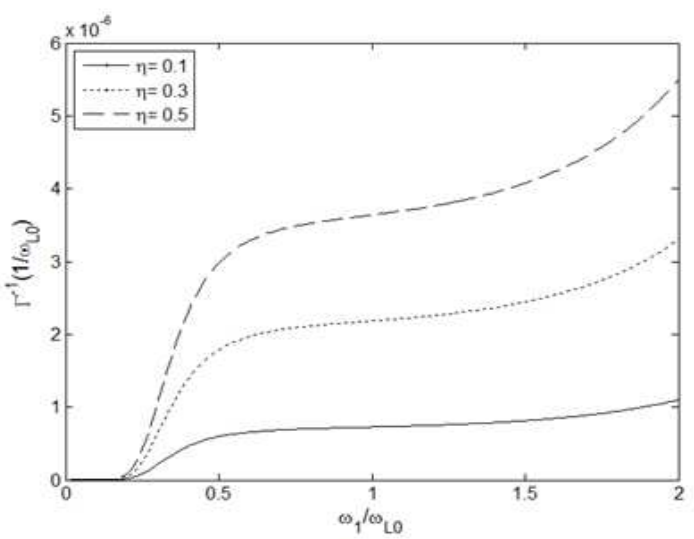

(a)

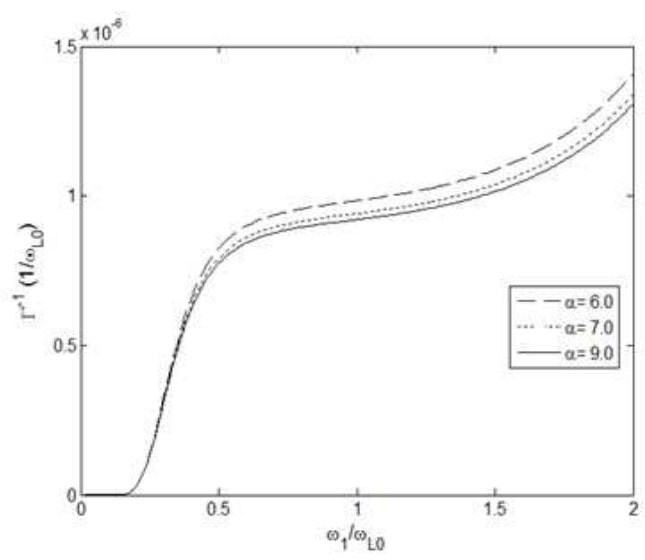

(b)

Figure 7. Decoherence time as a function of the confinement strength in the $x-y$-plane for (a) $l_{2}=0.45 ; \alpha=7.0, F=250.0$ and $\omega_{c}=10.0$ (b) $l_{2}=0.45 ; \eta=0.2, F=250.0$ and $\omega_{c}=10.0$.

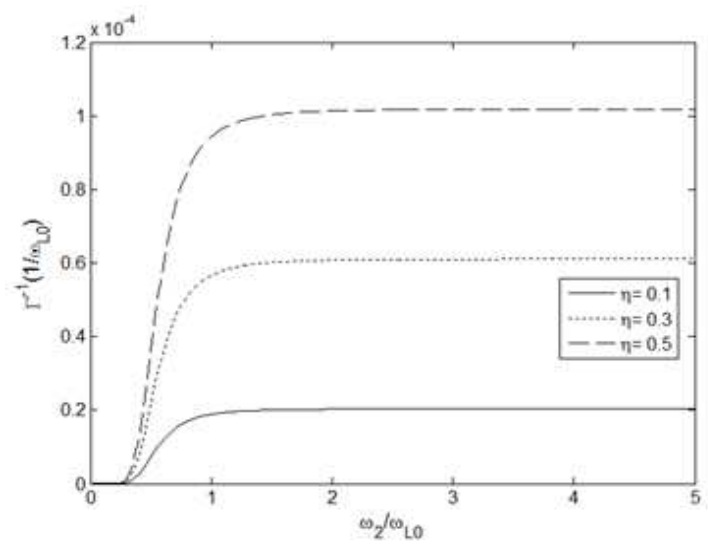

(a)

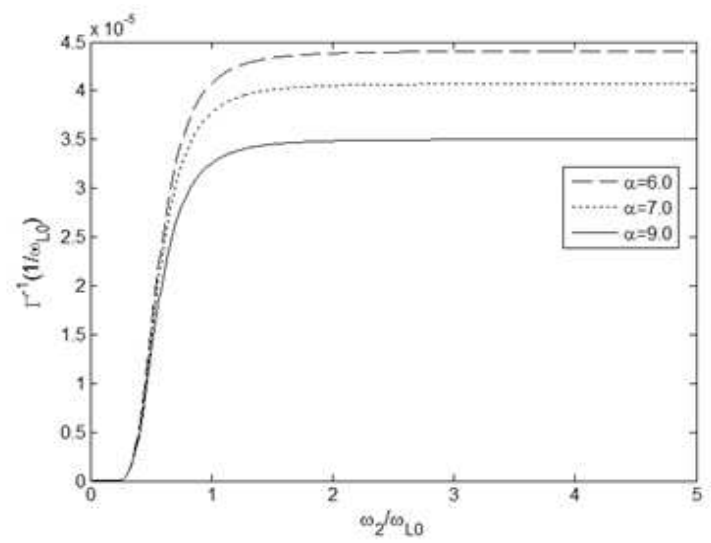

(b)

Figure 8. Decoherence time as a function of the confinement strength in the $z$ - direction for (a) $l_{1}=0.35 ; \alpha=7.0, F=250.0$ and $\omega_{c}=10.0$ (b) $l_{1}=0.35 ; \eta=0.2, F=250.0$ and $\omega_{c}=10.0$.

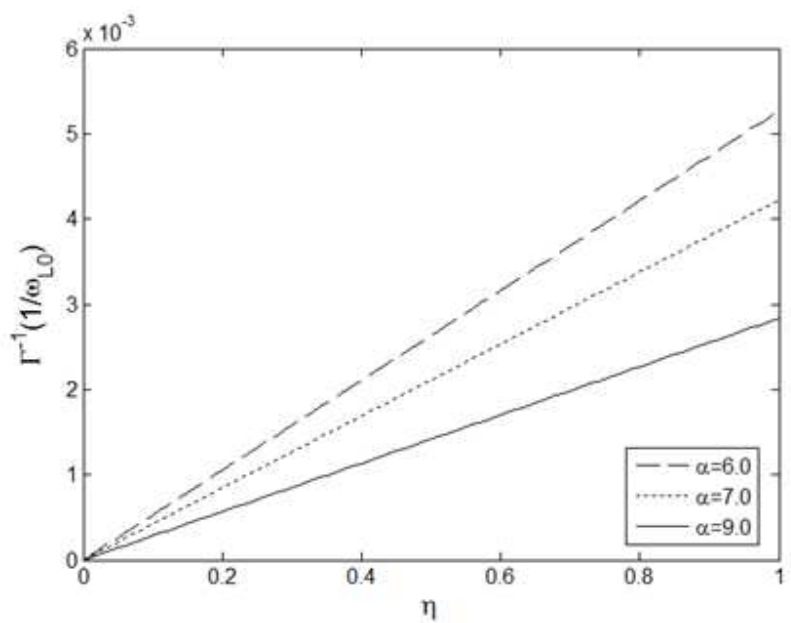

Figure 9. Decoherence time as a function of the confinement strength in the $x-y-$ plane for $l_{1}=0.25 ; l_{2}=0.35 ; \eta=0.2, F=250.0$ and $\omega_{c}=15.0$. 


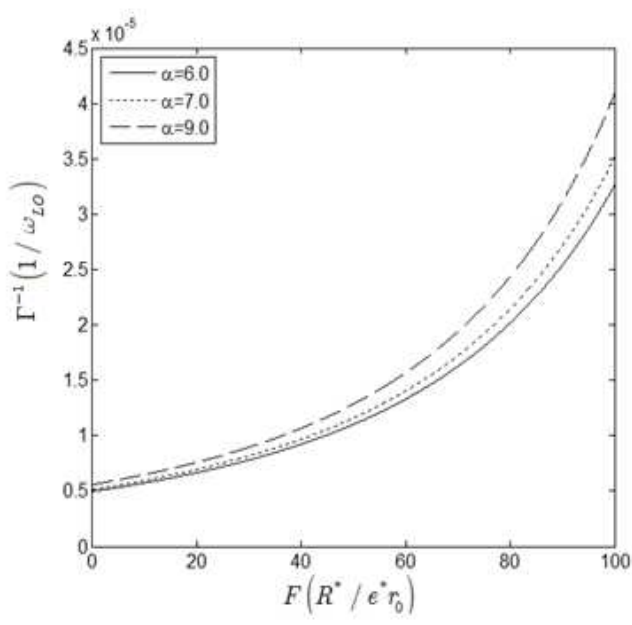

(a)

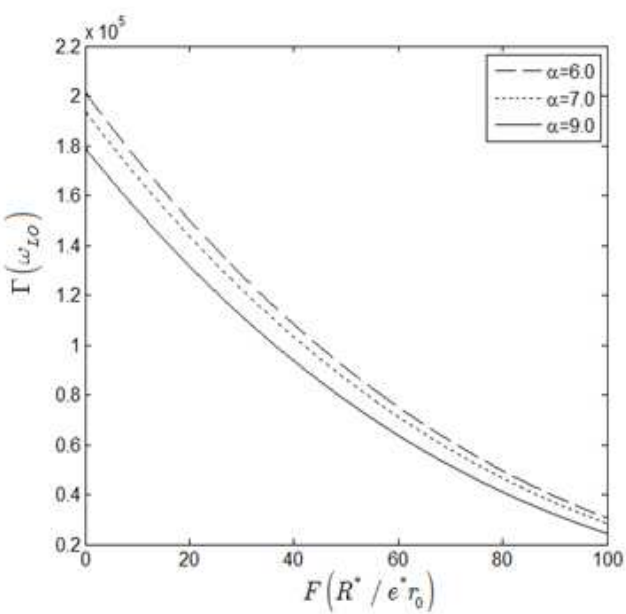

(b)

Figure 10. (a) Decoherence time as a function of the electric field strength for $l_{1}=0.25 ; l_{2}=0.35 ; \eta=0.2$, and $\omega_{c}=15.0$ (b) Spontaneous emission rate as a function of the electric field strength for $l_{1}=0.25 ; l_{2}=0.35 ; \eta=0.2$, and $\omega_{c}=15.0$.

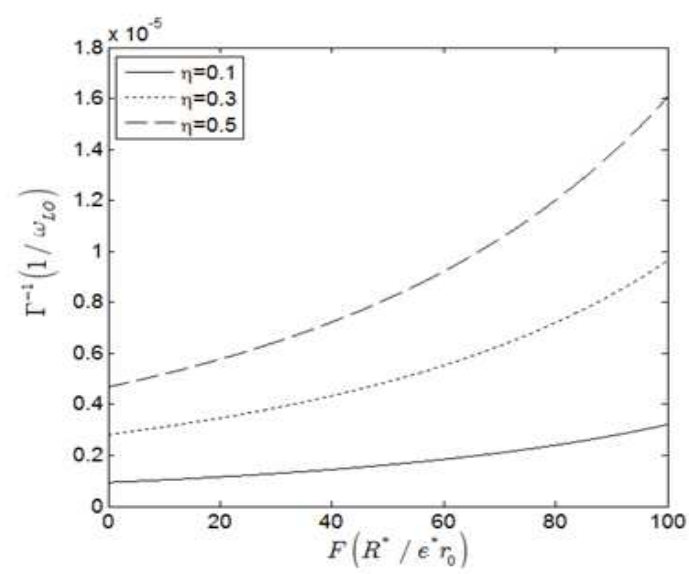

(a)

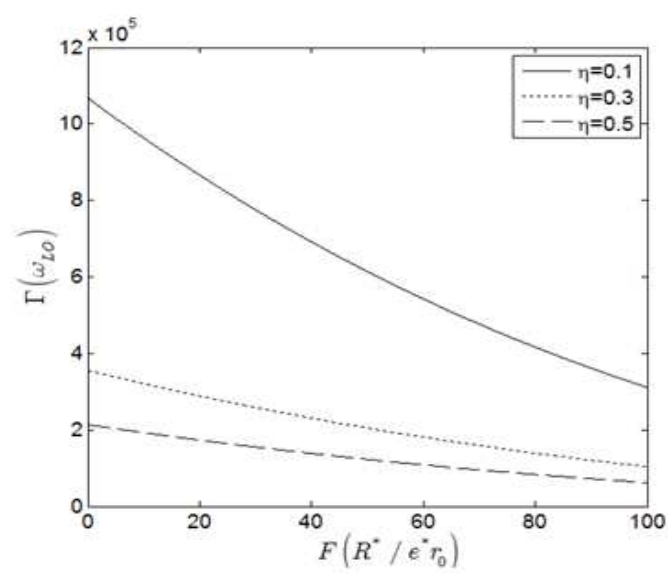

(b)

Figure 11. (a) Decoherence time as a function of the electric field strength for $l_{1}=0.25 ; l_{2}=0.35 ; \alpha=7.0$, and $\omega_{c}=15.0$ (b) Spontaneous emission rate as a function of the electric field strength for $l_{1}=0.25 ; l_{2}=0.35 ; \alpha=7.0$, and $\omega_{c}=15.0$.

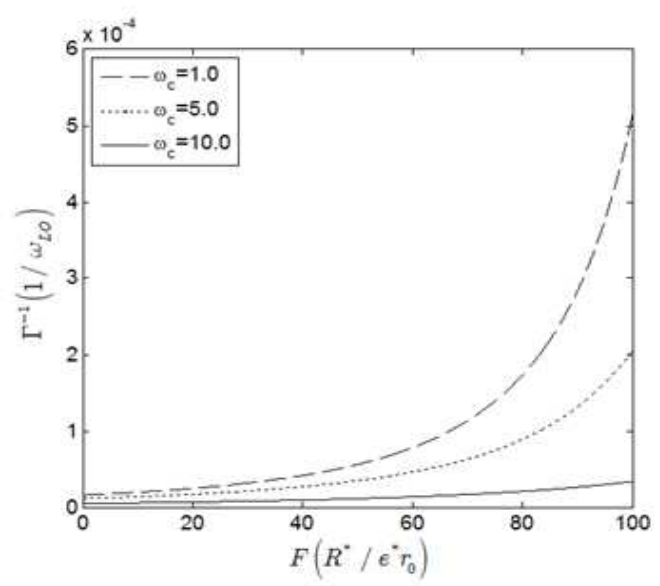

(a)

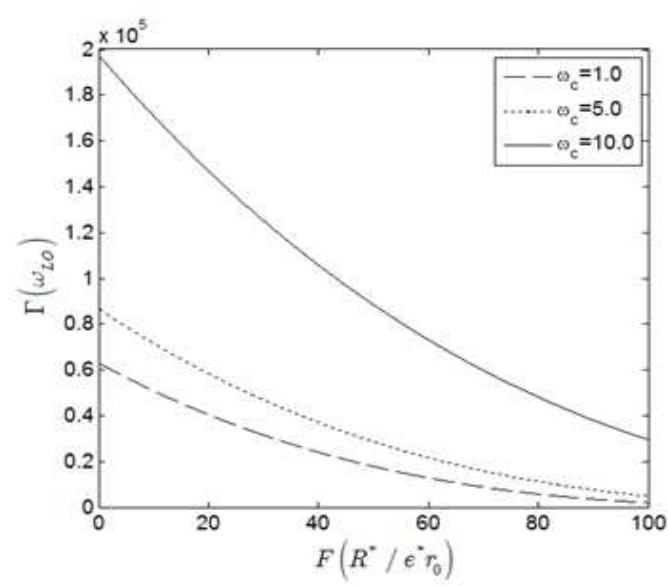

(b)

Figure 12. (a) Decoherence time as a function of the electric field strength for $l_{1}=0.25 ; l_{2}=0.35 ; \eta=0.2$ and $\alpha=7.0$ (b) Spontaneous emission rate as a function of electric field strength for $l_{1}=0.25 ; l_{2}=0.35 ; \eta=0.2$ and $\alpha=7.0$. 


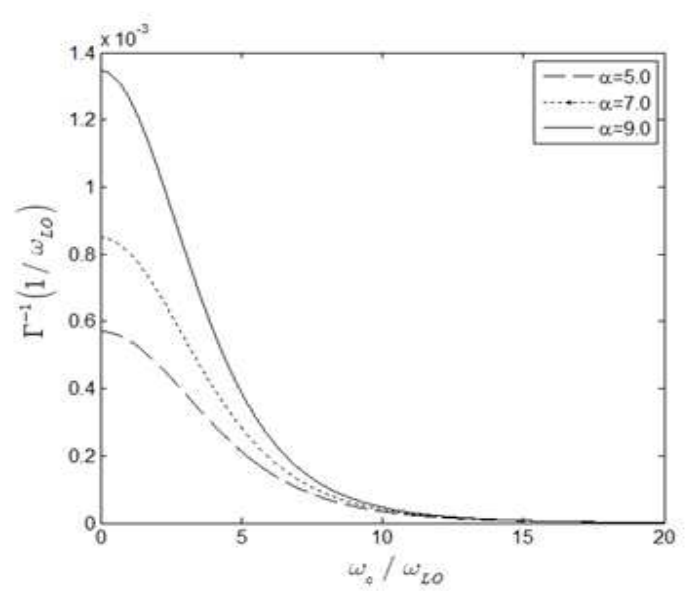

(a)

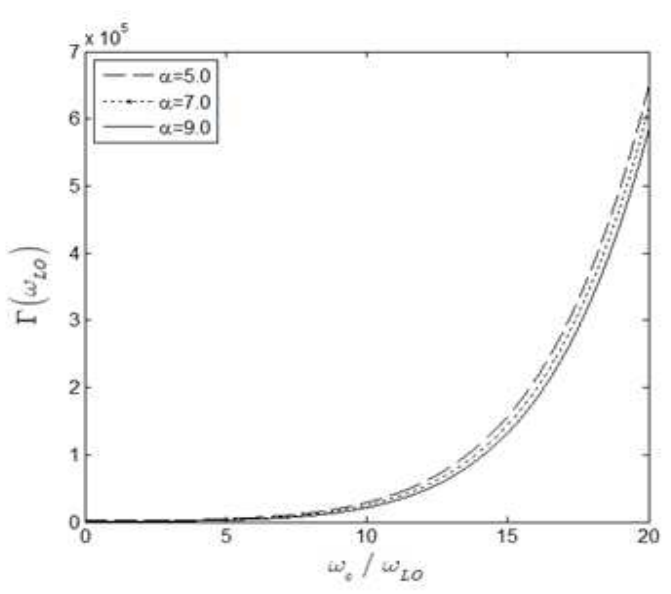

(b)

Figure 13. (a) Decoherence time as a function of the cyclotron frequency for $l_{1}=0.25 ; l_{2}=0.35 ; \eta=0.2 ; F=105.0$ (b) Spontaneous emission rate as a function the cyclotron frequency for $l_{1}=0.25 ; l_{2}=0.35 ; \eta=0.2 ; F=105.0$.

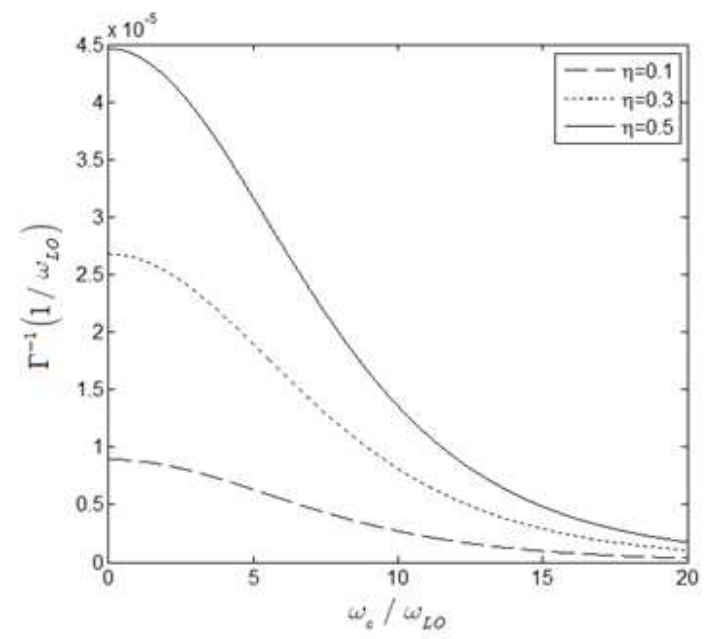

(a)

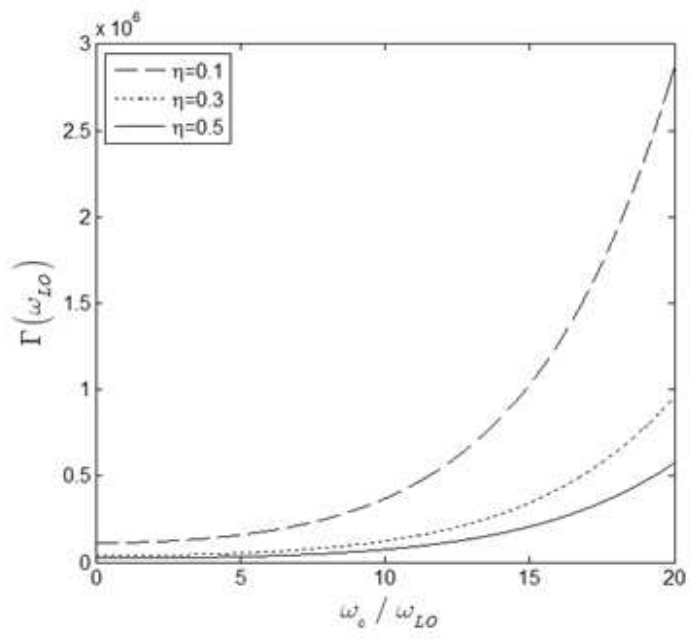

(b)

Figure 14. (a) Decoherence time as a function of the cyclotron frequency for $l_{1}=0.25 ; l_{2}=0.35 ; \alpha=7.0 ; F=105.0$ (b) Spontaneous emission rate as a function the cyclotron frequency for $l_{1}=0.25 ; l_{2}=0.35 ; \alpha=7.0 ; F=105.0$.

In figure 1, we have plotted the probability density when the electron is in the superposition state $|0\rangle$ and $|1\rangle$ for several different values of the cyclotron frequency (a) $\omega_{c}=2.7$; (b) $\omega_{c}=5.5$; (c) $\omega_{c}=10.5$ and the constant parameters $l_{1}=0.75 ; l_{2}=0.5 ; t=1.0 ; F=1.0 ; \alpha=7.0 ; \eta=0.2$ These figures show that the density is a decreasing function of cyclotron frequency.

In figure 2 , we have plotted the probability density when the electron is in the superposition state $|0\rangle$ and $|1\rangle$ for several different values of the electric field strength (a) $F=1.5$; (b) $F=3.5$; (c) $F=15.5$ and the constant parameters The probability is an increasing function of the electric field strength. The probability density of the electron oscillates with a period of oscillation $\tau=\frac{\hbar}{\left(E_{1}-E_{0}\right)}$ which shows a transfer of information from one state to another.

In figure 3, we have plotted the entropy as a function of time for several different values of the cyclotron frequency ( $\omega_{c}=1.0$ (fig. 3a); $\omega_{c}=15.0$ (fig. $3 \mathrm{~b}$ ); $\omega_{c}=50.0$ (fig. $3 \mathrm{c}$ ) and $\omega_{c}=100.0 \quad$ (fig. $\left.\left.3 \mathrm{~d}\right)\right)$ and the constant parameters $l_{1}=0.75 ; l_{2}=0.5 ; F=1.0 ; \alpha=7.0 ; \eta=0.2$.

In figure 4 , we have plotted the entropy as a function of time for several different values of the electric field strength ( $F=2.0$ (fig.4a); $F=10.0$ (fig. $4 b$ ); $F=20.0$ (fig.4c) and $F=30.0 \quad$ (fig.4d)) for constant $l_{1}=0.75 ; l_{2}=0.5 ; F=1.0 ; \alpha=7.0 ; \eta=0.2$. These figures show a decrease of the entropy with the increase of the cyclotron frequency (fig.5) and the increase of entropy with the increase of the electric field strength. The control of the coherence of the system can be done with the modulation of the electric and magnetic fields [18] 
In figure 5, we have plotted the density matrix elements $p_{01}$ as a function of time $\tau$ for $l_{1}=0.8 ; l_{2}=0.75 ; \alpha=7.0, F=1.5$ and $\omega_{c}=2.0$. It can be seen that the matrix element is a decreasing, non-periodic function of time. In the first pseudo period of oscillation, it is a decreasing function of the phonon dispersion coefficient while in the second, it is an increasing function of the same coefficient. The process continues till the annulation of the matrix element. From this result, to get the quantum dot with a longer decoherence time, we have to choose a material with a higher coefficient of phonon dispersion.

In figure 6 , we have plotted the density matrix elements $p_{01}$ as a function of time, $\tau$ for $l_{1}=0.8 ; l_{2}=0.75 ; \eta=0.2, F=1.5$ and $\omega_{c}=2.0$. It can be seen that the matrix element is a decreasing, non-periodic function of time. In the first pseudo period of oscillation, it is a decreasing function of the electron-LO- phonon coefficient and in the second, it is an increasing function of the same coefficient. This is because the electron-LO-phonon coupling constant is a decreasing function of the energy levels. When the energy between the ground and first excited state is increased, the decoherence time increases and when it is equal to the energy of emission of phonons, resonance occurs. This result is in agreement with those of Chen and Xiao JingLin [25], Yi-Fu Yu et al. [10] and Yong Sun et al. [24].

In figure 7 , we have plotted the decoherence time as a function of a confinement strength in the $x-y$-plane for $l_{2}=0.45 ; \alpha=7.0, F=250.0$ and $\omega_{c}=10.0 \quad$ (Fig. $\left.7 \mathrm{a}\right)$ and $l_{2}=0.45 ; \eta=0.2, F=250.0$ and $\omega_{c}=10.0$ (Fig. 7b). These figures show that the decoherence time is an increasing function of the confinement strength. The reason for this behavior is that, the decoherence time is inversely proportional to the energy spacing between the ground and first excited state; this energy decreases when the confinement strength increases and consequently, the decoherence time increases with the increase of the confinement strength in the $x-y$-plane. Fig.7a also shows that the decoherence time is an increasing function of the phonon dispersion coefficient $\eta$. From eq. 2.16, this behavior is justified because the decoherence time is proportional to this coefficient. This dispersion coefficient characterizes the material.

Fig. $7 b$ shows that the decoherence time is a decreasing function of the electron-phonon coupling constant. The electron-phonon coupling constant is weaker in the first excited state than in the ground state; from whence the energy gap between the ground and first excited state increases when the electron-phonon coupling constant increases. But this energy is inversely proportional to the decoherence time and this fact justifies the behavior of the decoherence time vis-à-vis the electron-phonon coupling constant. Resonance occurs when the energy gap equals the phonon emission and this energy gap causes increase of the spontaneous emission rate, consequently decreasing the decoherence time.

Figure 8 is a plot of the decoherence time as a function of the confinement strength in the $z-$ direction for $l_{1}=0.35 ; \alpha=7.0, F=250.0$ and $\omega_{c}=10.0$

$l_{1}=0.35 ; \eta=0.2, F=250.0$ and $\omega_{c}=10.0$

(Fig. 8a) and

(Fig.8b). The decoherence time increases with the increase of the confinement strength. This is because the energy spacing between the ground and first excited state is a decreasing function of the confinement strength. Fig. 8a also shows that the decoherence time is an increasing function of the phonon dispersion coefficient. The justification comes from equation (2.16) which shows the proportionality of the decoherence time to the phonon dispersion coefficient. Fig. $8 \mathrm{~b}$ shows that the decoherence time decreases with the increase of the electron-phonon coupling constant. The energy gap increases with the electron-phonon coupling constant and this justifies the fact that the decoherence time decreases with the increase of the electron-phonon coupling constant.

Figure 9 is a representation of the decoherence time as a function of the phonon dispersion coefficient in the $x-y-$ plane for $l_{1}=0.25 ; l_{2}=0.35 ; \eta=0.2, F=250.0$ and $\omega_{c}=15.0$. The figure shows that the decoherence time increases with the increase of the phonon dispersion coefficient, whereas it decreases with the increase of the electron-phonon coupling constant. The explanation of this behavior is the same as that in figs. 7 and 8. Because both the electron phonon coupling constant and the phonon dispersion coefficient are properties of the material, the choice of a material for the fabrication of a quantum dot has to be done very carefully.

Fig.10a is a plot of the decoherence time as a function of the electric field strength for $l_{1}=0.25 ; l_{2}=0.35 ; \eta=0.2$, and $\omega_{c}=15.0$. The decoherence time increase with the increase of the electric field strength. The electric field is considered as an external excitation source, which justifies the fact that in the presence of an electric field, the system quickly loses coherence. The figure also shows that the decoherence time decreases with the increase with the electron-phonon coupling constant. This is because the energy gap increases with the increase of the electron phonon coupling constant.

Fig. $10 \mathrm{~b}$ is a plot of the phonon emission rate as a function of the electric field strength for $l_{1}=0.25 ; l_{2}=0.35 ; \eta=0.2$, and $\omega_{c}=15.0$. This figure shows that the decoherence time decreases when the electric field strength increases and increases when the electron-phonon coupling strength decreases. The electric field causes the spontaneous emission of electrons. These results are in accordance with those of Fotue et $a l$. [26] and Y. Ji-Wen et $a l$. [27].

Fig. 11a is a plot of the decoherence time as a function of the electric field strength for $l_{1}=0.25 ; l_{2}=0.35 ; \alpha=7.0$, and $\omega_{c}=15.0$. The decoherence time increases with the increase of the electric field strength. The electric field is considered as an external excitation source, which justifies the fact that in the presence of an electric field the system quickly loses coherence.The figure also shows that the decoherence time increases with the increase of the phonon dispersion coefficient.

Fig. $11 \mathrm{~b}$ is the plot of the phonon emission rate as a function of electric field strength for 
$l_{1}=0.25 ; l_{2}=0.35 ; \alpha=7.0$, and $\omega_{c}=15.0$. This figure shows that the decoherence time decreases when the electric field strength increases and increases when the phonon dispersion coefficient decreases. The electric filed decay causes the spontaneous emission of electrons and the emission rate is highest at resonance.

Fig. 12a is a plot of the decoherence time as a function of the electric field strength for $l_{1}=0.25 ; l_{2}=0.35 ; \eta=0.2 ; \alpha=7.0$. The decoherence time increases with the increase of the electric field strength. The electric field is considered as an external excitation source, which justifies the fact that in the presence of an electric field the system quickly loses coherence. The figure also shows that the decoherence time decreases with the increase of the cyclotron frequency. The magnetic field increases the confinement of the electron while the electric field increases the rate of decoherence in the system[3, 19, 25].

Fig. $12 \mathrm{~b}$ is a plot of the phonon emission rate as a function of the electric field strength for $l_{1}=0.25 ; l_{2}=0.35 ; \eta=0.2 ; \alpha=7.0$. This figure shows that the decoherence time decreases when the electric field strength increases whereas it increases when the cyclotron frequency increases. The electric causes the spontaneous emission of electrons and the emission rate is highest at resonance.

Fig. 13a is a plot of the decoherence time as a function of the cyclotron frequency for $l_{1}=0.25 ; l_{2}=0.35 ; \eta=0.2 ; F=105.0$. This plot shows that the decoherence time decreases when the cyclotron frequency increases whereas it increases when the electron phonon coupling constant increases. The magnetic field enhances electron-phonon interaction and can thus be considered as a new confinement of the electron. As the magnetic field increases, the electron moves away from the center and gets closer to the surface along the axis, resulting in the contribution of the bulk LO phonon to the reduction of the ground state energy which is experimentally very important for the control and modulation of the intensity of optoelectronic devices [28-32].

Fig. $13 \mathrm{~b}$ is a plot of the spontaneous emission rate as a function of the cyclotron frequency for $l_{1}=0.25 ; l_{2}=0.35 ; \eta=0.2 ; F=105.0$. This plot shows that the spontaneous emission rate increases with the increase of the cyclotron frequency and decreases with the increases of the electron-phonon coupling constant. The presence of the magnetic field increases the emission rate [26].

In figure $14 \mathrm{a}$, we have plotted the decoherence time as a function of the cyclotron frequency for $l_{1}=0.25 ; l_{2}=0.35 ; \alpha=7.0 ; F=105.0$. This figure shows that the decoherence time decreases when the cyclotron frequency increases, whereas it increases when the phonon emission coefficient increases.

Fig. 14b is a numerical representation of the spontaneous emission rate as a function of the cyclotron frequency for $l_{1}=0.25 ; l_{2}=0.35 ; \alpha=7.0 ; F=105.0$. This plot shows that the spontaneous emission rate increases as the cyclotron frequency increases and as the phonon dispersion coefficient decreases $[19,25]$.

\section{Conclusion}

In this paper, we have derived the ground and first excited state energies of the polaron in an asymmetric cylindrical quantum dot and their relevant eigen-functions using the Pekar variational method. The single qubit can be envisaged as this kind of two-level quantum system in a QD. The probability density of the electron oscillates with a given period when the polaron is in the superposition of the ground and the first-excited states. The probability density of the polaron increases with a decrease of the magnetic field strength and increases with the electric field strength in the superposition state. The entropy is an increasing function of electric field and a decreasing function of the magnetic field strength. The coherence of the system can be controlled by tunneling the electric and magnetic fields. Because the electron phonon coupling constant and the phonon dispersion coefficient characterize the material, it is important to properly choose the material to use when fabricating the quantum dot. Our results should be meaningful for for the design and implementation of quantum computers both theoretically and experimentally and also for the control of decoherence in quantum systems.

\section{Acknowledgements}

We acknowledge the support of the Mesoscopic and Multilayers Structures Laboratory, Department of Physics, Faculty of Science where this work was carried out. We also thank the reviewers for their contributions.

\section{References}

[1] Li W. P., Yin J. W., Yu Y. F., Wang Z.W. and Xiao J.L., J. Low Temp Phys. 160, 112(2010).

[2] Yu Y. F., Li W. P., Yin J. W. and Xiao J.L., J. Low Temp. Phys. 50, 3322(2011)

[3] J.W. Yin, J.L. Xiao, Y.F. Yu, Z.W. Wang, Chin. Phys. B 18, 446(2009).

[4] Jia-kui S., Hong-Juan L., Jing-Lin X., Physica B 404,1961(2009).

[5] Nielsen M.A., Chang I. L., Quantum Computation and Quantum Information (Cambridge University Press, Cambridge, 2000).

[6] I. D’Amico, Microelectron. J. 37, 1440 (2006)

[7] Bennett C.H., DiVincenzo D.P., Nature 404, 247 (2000)

[8] Hawrylak P., Korkusinski M., Solid State Commun. 136, 508 (2005)

[9] Sarma S.D., Sousa R.D., Hu X.D., Koiller B., Solid State Commun. 133, 737 (2005) 
[10] Yi-Fu Yu, Wei-Ping Li, Ji-Wen Yin, Jing-Lin Xiao, Int. J. Theor. Phys 50, 3322(2011).

[11] Barnes, J.P., Warren, W.S., Phys. Rev. A 60, 4363 (1999)

[12] Tolkunov, D., Privman, V., Phys. Rev. A 69, 062309 (2004)

[13] Grodecka, A., Machnikowski, P., Phys. Rev. B 73, 125306 (2006)

[14] Lovric, M., Krojanski, H.G., Suter, D., Phys. Rev. A 75, 042305 (2007)

[15] Shannon CE. Bell Syst. Tech. J., 27(1), 379(1948)

[16] Sagar RP, Hô M, Mex J. , Chem. Soc. 52(1),60-66(2008).

[17] Guo-Hua Sun, Shi-Hai Dong. Phys. Scr. 87(4), 045003(2013)

[18] L. C. Fai, M. Tchoffo, J. T. Diffo and G. C. Fouokeng, Physical Review \& Research International 4(2): 267(2014)

[19] Jing-Lin Xiao, J. Low Temp Phys. 172(1-2), 122 (2013).

[20] Li S.S., Xia J.B., Yang F.H., Niu Z.C., Feng S.L., Zheng H.Z., J. Appl. Phys. 90, 6151 (2001)

[21] Li S.S.,Gui- Lu L., Bai F.S., Feng S.L., Zheng H.Z., Proc. Natl. Acad. Sci. USA 98, 11847 (2001)

[22] Wang Z.W., Xiao J.L., Acta Phys. Sin. 56, 678 (2007)
[23] L.D Landau, E.M. Lifshitz, Quantum Mechanics (Non relativistic Theory) (Pergamon, London, 1987)

[24] Yong Sun, Zhao-Hua Ding and Jing-Lin Xiao, J. Low temp. Phys. 177, 151(2014)

[25] Chen Shihua and Xiao Jing-Lin, Chinese Journal of Electronics 18(2), 2009

[26] A. J. Fotue, S. C. Kenfack, H. Fotsin, M. Tiotsop, L. C. Fai and M.P. Tabue Djemmo, Physical Science international journal 6(1), 15(2015).

[27] Y. Ji-Wen , X. Jing-Lin, Y. Yi-Fu and W. Zi-Wu, Chinese Physics B 18(2), 446(2009)

[28] B.S. Kandemir, A. Cetin, J. Phys. Condens. Matter 17(4), $667(2003)$

[29] V. L. Nguyen, M. T. Nguyen and T. D. Nguyen, Physica B 292(1), 159(2000).

[30] C. S. Wang and J. L. Xiao, Mod. Phys. Lett. B 26, 1150003 (2012) [10 pages]

[31] Y. Lepine and G. Bruneau, J. Phys. Condens. Matter 10, 1495(1998).

[32] B. S. Kandemir and T. Altanhan, Eur. Phys. J. B. 33, 227(2003). 